\title{
On linear operators and functors extending pseudometrics
}

\author{
by
}

Czesław Bessaga (Warszawa)

\begin{abstract}
For some pairs $(X, A)$, where $X$ is a metrizable topological space and $A$ its closed subset, continuous, linear (i.e., additive and positive-homogeneous) operators extending metrics for $A$ to metrics for $X$ are constructed. They are defined by explicit analytic formulas, and also regarded as functors between certain categories. An essential role is played by "squeezed cones" related to the classical cone construction. The main result: if $A$ is a nondegenerate absolute neighborhood retract for metric spaces, then continuous linear operators extending metrics always exist.
\end{abstract}

There are two roots for the present study. The first is the theorem of Hausdorff $[\mathrm{H}]$ on extending metrics for a closed subset $A$ of a metrizable topological space $X$ to metrics on the whole space $X$. The second is the Borsuk-Dugundji theorem ([Bo], $[\mathrm{Du}])$ on the existence of continuous linear operators extending continuous functions on $A$ to continuous functions on $X$. The author tried to prove the existence of continuous, linear (i.e., additive and positive-homogeneous) operators extending admissible metrics from $A$ to $X$, and has only succeeded in special situations, e.g., when $A$ is a nondegenerate ANR for metric spaces (Corollary 2.3). Very stimulating and helpful for our discussion was the paper [KN] of Nguyen Van Khue and Nguyen To Nhu: they were the first to construct continuous (but merely sublinear) operators extending metrics.

The main part of the paper are $\S \S 1$ and 2 which lead to the abovementioned Corollary 2.3. A crucial role is played by an extension of spaces and pseudometrics called the squeezed cone construction (in symbols: sc) which is related to the classical cone construction in topology. $\S 3$ contains examples of spaces with an "absolute" property of linear extending of metrics but lacking the ANR property. $\S 4$ is devoted to discussion of the linear extension constructions viewed as functors between certain categories. Finally, in $\S 5$ we ask some questions, mostly functional-analytic, related to the results, proofs and the philosophy of the paper; also some comments are included. 
It should be noted that the extension operators defined in this paper are expressed by explicit analytic formulas and therefore they can easily be examined for additional properties, e.g., preservation of special classes of metrics.

Information on the history of the subject of extensions of metrics (till the late seventies), interesting original results on extending individual metrics, and a strengthening of Hausdorff's theorem can be found in Sect. 6 of Luukkainen's paper $[\mathrm{L}]$, and in the papers listed in the bibliography of $[\mathrm{L}]$, in particular in $[\mathrm{KN}],[\mathrm{N}]$ and $[\mathrm{T}]$.

Acknowledgement. I want to acknowledge my indebtedness to Mr. Lauri Leppik, the discussion with whom in 1988 (when he was a student of Tallin State Pedagogical Institute, visiting Warsaw University) has helped to crystallize the concept of squeezed cone. I am also very grateful to the referee who studied very carefully the previous versions of the manuscript, pointed out numerous misprints and some unprecise, or even incorrect statements and made valuable suggestions improving the paper.

\section{Squeezed products of spaces and pseudometrics}

1.1. Consider two pointed sets $(A, O)$ and $(T, O)$, i.e., $A$ and $T$ are arbitrary nonempty sets and $o \in A, O \in T$ their base points. Let

$$
A * T=(A \times T) /(A \times\{O\} \cup\{o\} \times T) ;
$$

the set $A \times\{O\} \cup\{o\} \times T$ is regarded as a single point $\mathbf{0} \in A * T$, and consequently we write

$$
(a, u)=\mathbf{0} \quad \text { iff } \quad a=o \text { or } u=O .
$$

Definition. The set $A * T$ is called the squeezed product of $A$ and $T$ determined by the base points $o$ and $O$.

By a symmetric function on $X \times X$ we mean a function $p: X \times X \rightarrow \mathbb{R}$ satisfying $p(x, y)=p(y, x), p(x, x)=0$. The linear space of symmetric functions on $X \times X$ will be denoted by $\mathrm{S}(X)$; the symbols $\mathrm{P}(X)$ and $\mathrm{M}(X)$ will stand for its subsets (cones) consisting of pseudometrics and metrics, respectively. Let

$$
\|p\|=\sup \{|p(x, y)|: x, y \in X\} \quad \text { for } p: X \times X \rightarrow \mathbb{R} .
$$

Assuming $p \in \mathrm{S}(A), \delta \in \mathrm{S}(T), a, b \in A, u, v \in T$, we define

$$
\begin{aligned}
p * \delta((a, u),(b, v))= & 2^{-1}[p(a, b)(\delta(u, O)+\delta(v, O)-\delta(u, v)) \\
& +p(a, o)(\delta(u, O)-\delta(v, O)+\delta(u, v)) \\
& +p(b, o)(\delta(v, O)-\delta(u, O)+\delta(u, v))] .
\end{aligned}
$$

Remark. A routine calculation yields

$$
p * \delta((a, u),(b, v))=p * \delta((b, v),(a, u))=\delta * p((u, a),(v, b)),
$$


(14) if $(b, v)=\mathbf{0}$, then $p * \delta((a, u),(b, v))=p(a, o) \delta(u, O)$,

(15) $p * \delta((a, u),(b, u))=p(a, b) \delta(u, O)$,

for every $a, b \in A, u, v \in T$.

1.2. According to (13) and (14) the function $p * \delta$ may be regarded as a function

$$
p * \delta:(A * T) \times(A * T) \rightarrow \mathbb{R}, \quad p * \delta \in \mathrm{S}(A * T) .
$$

Definition. The function (21) is called the squeezed product of $p$ and $\delta$ determined by the base points $O$ and $O$.

Proposition. The operation $*: \mathrm{S}(A) \times \mathrm{S}(T) \rightarrow \mathrm{S}(A * T)$ is bilinear and has the following properties:

(22) $\quad\|p * \delta\| \leq 9 \cdot 2^{-1}\|p\| \cdot\|\delta\|$,

(23) $\quad|p * \delta((a, u),(b, v))| \leq 2^{-1}(|\delta(u, O)|+|\delta(v, O)|+|\delta(u, v)|)$

$$
\times(|p(a, o)|+|p(b, o)|+|p(a, b)|),
$$

(24) if $p \geq 0$ and $\delta \in \mathrm{P}(T)$, then $p * \delta \geq 0$,

(25) $|p * \delta((a, u), \mathbf{0})| \leq \min [|p(a, o)|\|\delta\|,|\delta(u, O)|\|p\|]$.

If $p \in \mathrm{P}(A)$ and $\delta \in \mathrm{P}(T)$, then:

(26) $\quad p * \delta((a, u),(b, v)) \leq p(a, b) \delta(v, O)+\delta(u, v)(p(a, b)+2 p(b, o))$,

(27) $\quad p * \delta((a, u),(b, v)) \geq \delta(v, O) \cdot \min [p(a, b), p(b, o)]$,

$\left(27^{\prime}\right) \quad p * \delta((a, u),(b, v)) \geq p(b, o) \cdot \min [\delta(u, v), \delta(v, O)]$.

Proof. The estimates (22) and (23) are obvious. The estimate (25) follows directly from (14). Define

$$
\Delta=p * \delta((a, u),(b, v)) .
$$

Proof of (24). If $p \geq 0$ and $\delta \in \mathrm{P}(T)$, then all the three terms on the right-hand side of (12) are nonnegative, and therefore $\Delta \geq 0$.

Pro of of (26). By (12) and the triangle inequality,

$$
\begin{aligned}
\Delta & \leq 2^{-1}[p(a, b) 2 \delta(v, O)+p(a, o) 2 \delta(u, v)+p(b, o) 2 \delta(u, v)] \\
& =p(a, b) \delta(v, O)+\delta(u, v)(p(a, o)+p(b, o)) \\
& \leq p(a, b) \delta(v, O)+\delta(u, v)(p(a, b)+2 p(b, o)) .
\end{aligned}
$$

Proof of (27). Let $\eta=\min [p(a, b), p(b, o)] / 2$. Ignoring the second term on the right-hand side of (12), we get

$\Delta \geq \eta(\delta(u, O)+\delta(v, O)-\delta(u, v)+\delta(v, O)-\delta(u, O)+\delta(u, v))=2 \eta \delta(v, O)$, which is the inequality (27).

The estimate $\left(27^{\prime}\right)$ follows from (27) and the symmetry condition (13). 
Theorem. If $p$ and $\delta$ are pseudometrics [metrics], then $p * \delta$ is a pseudometric [a metric].

Proof. Observe that, by (27), $\left(27^{\prime}\right)$ and (14), if $p$ and $\delta$ are metrics and if $(a, u) \neq(b, v)$ as points of $A * T$, then

$$
p * \delta((a, u),(b, v)) \neq 0 .
$$

Hence it is enough to prove the assertion concerning pseudometrics.

Begin with the special case:

$$
A=\{1,2,3, o\} .
$$

Let $H$ denote the class of nonempty subsets $B \subseteq\{1,2,3\}$. We say that the set $B \in H$ separates the pair $(a, b) \in A \times A$, and write $(a, b) \mid B$, if $B$ and $A \backslash B$ each contain one point of the pair. For each $B \in H$, consider the pseudometric $p_{B} \in \mathrm{P}(A)$ defined by

$$
p_{B}(a, b)= \begin{cases}1 & \text { if }(a, b) \mid B \\ 0 & \text { otherwise. }\end{cases}
$$

Fix $\delta \in \mathrm{P}(T)$, let $d_{B}=p_{B} * \delta$, and define $r_{B}: A * T \rightarrow T$ by

$$
r_{B}(a, u)= \begin{cases}u & \text { if } a \in B \\ O & \text { if } a \notin B .\end{cases}
$$

A direct calculation yields

$$
d_{B}((a, u),(b, v))=\delta\left(r_{B}(a, u), r_{B}(b, v)\right),
$$

whence it is seen that $d_{B} \in \mathrm{P}(A * T)$ for every $B \in H$. Therefore, by Proposition $1.2, p * \delta \in \mathrm{P}(A * T)$ if $p$ is an arbitrary nonnegative linear combination of the pseudometrics $p_{B}$. Hence, under the assumption (28), the theorem is a consequence of the following

Lemma. If $A=\{1,2,3, o\}$ and $H$ are as above, then every $p \in \mathrm{P}(A)$ is a nonnegative linear combination of the pseudometrics $p_{B}, B \in H$.

Proof. First, introduce the abbreviations: $p_{o}=p_{\{1,2,3\}}, p_{i}=p_{\{i\}}$ for $i \in\{1,2,3\}, p_{i j}=p_{\{i, j\}}$ for $\{i, j\} \subseteq\{1,2,3\}$.

Observe that if $p \in \mathrm{P}(A) \backslash \mathrm{M}(A)$, and for instance $p(o, 1)=0$, then

$$
\begin{aligned}
2 p= & {[p(1,2)+p(1,3)-p(2,3)] p_{23}+[p(2,1)+p(2,3)-p(1,3)] p_{2} } \\
& +[p(3,1)+p(3,2)-p(1,2)] p_{3},
\end{aligned}
$$

i.e., $p$ satisfies the assertion of the Lemma.

So, it is enough to consider the case where $p \in \mathrm{M}(A)$. Let us say that a point $c \in A$ is internal for $p$ if there exist $a, b \in A$ such that $0<p(a, c)<$ $p(a, b)$ and $p(a, b)=p(a, c)+p(b, c)$; define

$$
\mathrm{i}(p)=\{c \in A: c \text { is internal for } p\} .
$$


Assume that $c \in A \backslash \mathrm{i}(p)$ and $u=\sup \left\{t \geq 0: p-t p_{c} \in \mathrm{P}(A)\right\}$. It is easy to see that $q=p-u p_{c} \in \mathrm{P}(A)$ and that either $q \in \mathrm{P}(A) \backslash \mathrm{M}(A)$ or $q \in \mathrm{M}(A)$ with $\mathrm{i}(q)=\mathrm{i}(p) \cup\{c\}$. Hence, after subtracting from $p$ a nonnegative linear combination of the pseudometrics $p_{c}, c \in A$, we arrive at a pseudometric $q$ which either is not a metric or is a metric with $\mathrm{i}(q)=A$. The first case is already done. An elementary examination of the second case yields that after renaming the points $1,2,3, o$, if necessary, the only possible configuration is given by the equalities

$$
p(o, 1)=p(2,3)=\alpha, \quad p(o, 3)=p(1,2)=\beta, \quad p(o, 2)=p(1,3)=\alpha+\beta .
$$

Hence $p=\alpha p_{12}+\beta p_{23}$. This completes the proof of the Lemma.

Proof of the Theorem (cont.). If card $A \leq 3$, we may assume that $\{o\} \subseteq A \subseteq\{1,2,3, o\}$. Obviously, each $p \in \mathrm{P}(A)$ can be extended to a pseudometric $p_{0} \in \mathrm{P}(\{1,2,3, o\})$ and then $p * \delta$ is the restriction of $p_{0} * \delta$. This observation reduces the proof to the case (28).

Now assume that card $A>4$. The triangle inequality for $p * \delta$, to be checked, involves at most three distinct points of $A * T$. We denote the $A$-coordinates of these points by some of the symbols $1,2,3, o$, reserving the rest of them for denoting other points of $A$ (and remembering that $o$ is the base point of $A$ !). This way the proof again reduces to the case (28).

The statement (14) together with the estimates (26), (27) and $\left(27^{\prime}\right)$ yield the following description of the convergence with respect to the squeezed product of bounded pseudometrics:

Corollary. Assume that $p \in \mathrm{P}(A)$ and $\delta \in \mathrm{P}(T)$ are bounded. Then:

(i) $p * \delta\left(\left(a_{n}, u_{n}\right),(a, u)\right) \rightarrow 0$ iff $p\left(a_{n}, a\right) \rightarrow 0$ and $\delta\left(u_{n}, u\right) \rightarrow 0$, provided that $p(a, o) \delta(u, O) \neq 0$.

(ii) $p * \delta\left(\left(a_{n}, u_{n}\right),(a, u)\right) \rightarrow 0$ iff $p\left(a_{n}, o\right) \delta\left(u_{n}, O\right) \rightarrow 0$ iff $\min \left[p\left(a_{n}, o\right)\right.$, $\left.\delta\left(u_{n}, O\right)\right] \rightarrow 0$, provided that $p(a, o) \delta(u, O)=0$.

Let us note that the condition $p(a, o) \delta(u, O)=0$ means the same as $p * \delta((a, u), \mathbf{0})=0$.

1.3. Definition. Let $p, d \in \mathrm{P}(X)$. We say that $d$ dominates $p$ if $d\left(x_{n}, x\right) \rightarrow 0$ implies $p\left(x_{n}, x\right) \rightarrow 0$, and $p, d$ are said to be equivalent, written $p \simeq d$, if they dominate each other.

Theorem. If $p, q \in \mathrm{P}(A), \delta \in \mathrm{P}(T),\|p\|<\infty$ and $\|\delta\|<\infty$ and $q$ dominates $p$, then $q * \delta$ dominates $p * \delta$. In particular, if all three pseudometrics $p, q, \delta$ are bounded and $p \simeq q$, then $p * \delta \simeq q * \delta$.

Proof. Let $s=\min [q, 1]$. By $(12), q * \delta \geq s * \delta$, in particular, $q * \delta$ dominates $s * \delta$. Therefore, without loss of generality we may assume that 
$\|q\|<\infty$. In this case, the assertion of the Theorem follows from Corollary 1.2 .

1.4. Assume that $A, T, X, \ldots$ are metrizable topological spaces, and $o \in A, O \in T$ their base points. A metric $\delta \in \mathrm{M}(X)$ is said to be admissible if it determines the topology of $X$, and dominating if it dominates the admissible metrics.

Denote by

$$
\operatorname{Pb}(X), \quad \operatorname{Pc}(X), \quad \operatorname{Pbc}(X)
$$

the sets of all bounded, continuous, bounded and continuous pseudometrics for $X$, respectively, and denote by

$$
\operatorname{Mb}(X), \quad \operatorname{Mc}(X), \quad \operatorname{Mbc}(X), \quad \operatorname{Md}(X), \quad \operatorname{Ma}(X), \quad \operatorname{Mab}(X)
$$

the sets of all bounded, continuous, bounded and continuous, dominating, admissible, admissible and bounded metrics for $X$, respectively.

From the last theorem it follows that any choice of the metrics $p \in$ $\operatorname{Mab}(A)$ and $\delta \in \operatorname{Mab}(T)$ gives rise to the same topology of $A * T$ induced by $p * \delta$. This motivates the following:

Definition. The squeezed product of metrizable topological spaces $A$, $T$ is $A * T$ equipped with the topology induced by $p * \delta$ where $p \in \operatorname{Mab}(A)$, $\delta \in \operatorname{Mab}(T)$.

According to the last Definition and to Theorem 1.3, we have

TheOREM. Let $\mathrm{K}$ denote one of the symbols $\mathrm{Pbc}, \mathrm{Mbc}, \mathrm{Md}$, Mab. Then $p \in \mathrm{K}(A), \delta \in \mathrm{K}(T)$ implies $p * \delta \in \mathrm{K}(A * T)$.

Rem ark. From formula (14) it follows that if $p$ and $\delta$ are continuous, $p$ is unbounded, $\delta$ is a metric, and $O$ is not an isolated point of $T$, then $p * \delta$ is not continuous at $\mathbf{0}$.

Corollary 1.2 yields

Proposition. Convergence in $A * T$ is characterized as follows:

(i) $\left(a_{n}, u_{n}\right) \rightarrow(a, u) \neq \mathbf{0}$ iff $a_{n} \rightarrow a$ and $u_{n} \rightarrow u$;

(ii) $\left(a_{n}, u_{n}\right) \rightarrow \mathbf{0}$ iff either $a_{n} \rightarrow$ o or $u_{n} \rightarrow O$ or the set $\mathbb{N}$ splits into two subsequences $(k(n))$ and $(l(n))$ such that $a_{k(n)} \rightarrow o$ and $u_{l(n)} \rightarrow O$.

Hint: If $\mathbb{N}^{\prime}=\left\{n \in \mathbb{N}: p\left(a_{n}, o\right)<\delta\left(u_{n}, O\right)\right\}$ and $\mathbb{N} \backslash \mathbb{N}^{\prime}$ are both infinite, we arrange the elements of these sets into the subsequences $(k(n))$ and $(l(n))$, respectively.

Let us observe that the topology of $A * T$ is determined by all open subsets of $(A \backslash\{o\}) \times(T \backslash\{O\})$ and by all sets of the form $U \times T \cup A \times V$ where $U$ is an open neighborhood of $o$ in $A$ and $V$ is an open neighborhood of $O$ in $T$. 
2. Squeezed cones. Linear extending of metrics from neighborhood retracts. The symbols $A, B, X, Y, Z, \ldots$ will stand for metrizable topological spaces.

2.1. Let $A$ be a space with base point $o$, and let $\mathbf{I}$ be the segment $[0 ; 1]$ with base point 0 equipped with the standard metric: $s(u, v)=|u-v|$.

Definition. The squeezed cone over $(A, o)$ is the space $\operatorname{sc}(A, o)=A * \mathbf{I}$, denoted also briefly by $\operatorname{sc} A$; for any $p \in \mathrm{S}(A)$, we let

$$
\begin{aligned}
\operatorname{sc} p((a, u),(b, v))= & 2^{-1}[p(a, b)(u+v-|u-v|) \\
& +p(a, o)(u-v+|u-v|) \\
& +p(b, o)(v-u+|u-v|)] .
\end{aligned}
$$

The space $A$ itself is regarded as the subset $A \times\{1\}$ (more precisely: $(A \backslash$ $\{o\}) \times\{1\} \cup\{\mathbf{0}\})$. The identification is justified by the assertion (11) below.

Proposition 1.4 yields

Proposition. Convergence in sc $A$ is characterized as follows:

(i) $\left(a_{n}, u_{n}\right) \rightarrow(a, u) \neq \mathbf{0}$ iff $a_{n} \rightarrow a$ and $u_{n} \rightarrow u$;

(ii) $\left(a_{n}, u_{n}\right) \rightarrow \mathbf{0}$ iff either $a_{n} \rightarrow$ o or $u_{n} \rightarrow 0$ or $\mathbb{N}$ splits into two subsequences $(k(n))$ and $(l(n))$ such that $a_{k(n)} \rightarrow o$ and $u_{l(n)} \rightarrow 0$.

The next theorem is related to Proposition 1.2.

Theorem. The map sc $: \mathrm{S}(A) \rightarrow \mathrm{S}(\mathrm{sc} A)$ is a linear operator. For every $a, b \in A, u, v \in \mathbf{I}, p, q \in \mathrm{S}(A)$, we have

(11) $\operatorname{sc} p((a, u),(b, u))=p(a, b) u ; \operatorname{sc} p((a, u),(a, v))=p(a, o)|u-v|$,

(12) $\operatorname{sc} p((a, u), \mathbf{0})=p(a, o) u$,

(13) $\|\operatorname{sc} p\| \leq\|p\|$; if $p \leq q$, then $\operatorname{sc} p \leq \operatorname{sc} q$,

(14) if $\mathrm{K}$ is one of the symbols $\mathrm{P}, \mathrm{M}, \mathrm{Pbc}, \mathrm{Mbc}, \mathrm{Md}$, Mab, then $p \in \mathrm{K}(A)$ implies $\operatorname{sc} p \in \mathrm{K}(\operatorname{sc} A)$.

2.2. Recall the following

Definition. A closed subset $A$ of $X$ is said to be an NR (neighborhood retract) of $X$ if there is a continuous retraction $\beta$ from an open neighborhood $U$ of $A$ in $X$ onto $A$; a space $Y$ is an ANR (absolute neighborhood retract) if it is an NR of every space $Z$ containing $Y$ as a closed subset.

Proposition. If $A$ is an $\mathrm{NR}$ of $X$, then there is a continuous retraction $r$ of $\operatorname{sc} X$ onto sc $A$ such that, for every $p \in \operatorname{Pc}(A)$, the pseudometric $F p \in$ $\mathrm{P}(X)$ defined by

$$
F p(x, y)=\operatorname{sc} p(r x, r y) \quad \text { for } x, y \in X
$$

is continuous and, evidently, extends $p$. 
We note that, if $p$ is unbounded, the formula (21), regarded for $x, y \in$ sc $X$, yields a discontinuous pseudometric (cf. Remark 1.4).

Proof. Let $U$ and $\beta$ be those of the Definition. Assume $V$ to be an open neighborhood of $A$ such that the closure of $V$ is in $U$, and let $g: X \rightarrow \mathbf{I}$ be a continuous Urysohn function such that $g^{-1}(0)=X \backslash V$ and $g(a)=1$ for $a \in A$. The required retraction $r: \operatorname{sc} X \rightarrow \operatorname{sc} A$ is defined by

$$
r(x, u)=(\alpha(x), g(x) u),
$$

where $\alpha(x)=\beta(x)$ if $x \in U$ and $\alpha(x)=o$ if $x \in X \backslash U$.

Evidently, $r(x, y)=\mathbf{0}$ if $(x, u)=\mathbf{0}$, i.e., $r$ is well-defined on $\operatorname{sc} X$; it is also clear that $r(a, u)=(a, u)$ for $(a, u) \in \operatorname{sc} A$. Thus $r$ is a retraction.

During the proof we several times employ the fact that $\alpha$ is continuous at each point $x \in U$, and is equal to $o$ on $X \backslash U \cup\{o\}$, other properties of $\alpha, \beta$ and $g$ (stated in their definitions), and the characterization of convergence ((i) and (ii) of Proposition 2.1). We shall make no further specific reference to these facts.

For checking the continuity of $r$ assume that $\left(x_{n}, u_{n}\right) \rightarrow(x, u)$. We shall consider several cases separately.

Case 1: $(x, u) \neq \mathbf{0}$ and $x \in U$. Then $\alpha\left(x_{n}\right) \rightarrow \alpha(x)$ and $g\left(x_{n}\right) u_{n} \rightarrow$ $g(x) u$, i.e., $r\left(x_{n}, u_{n}\right) \rightarrow r(x, u)$.

Case 2: $(x, u) \neq \mathbf{0}$ and $x \in X \backslash U$. Then $g\left(x_{n}\right) \rightarrow g(x)=0$ and $g\left(x_{n}\right) u_{n} \rightarrow 0$. Hence $r\left(x_{n}, u_{n}\right) \rightarrow \mathbf{0}=r(x, u)$.

Case 3: $(x, u)=\mathbf{0}$. Then, according to Proposition 2.1, it is enough to restrict attention to the cases: $x_{n} \rightarrow o ; u_{n} \rightarrow 0$. In either, $r\left(x_{n}, u_{n}\right) \rightarrow \mathbf{0}=$ $r(\mathbf{0})$.

It remains to check the continuity of the pseudometric $F p$. Assume that $x, x_{n} \in X$ and $x_{n} \rightarrow x$. Let $r x=(a, u), r x_{n}=\left(a_{n}, u_{n}\right)$.

If $r x \neq \mathbf{0}$, then, by the continuity of $r$,

$$
p\left(a_{n}, a\right) \rightarrow 0 \text { and }\left|u_{n}-u\right| \rightarrow 0 .
$$

Hence, by (11)

$$
\begin{aligned}
F p\left(x_{n}, x\right) & =\operatorname{sc} p\left(\left(a_{n}, u_{n}\right),(a, u)\right) \\
& \leq \operatorname{sc} p\left(\left(a_{n}, u_{n}\right),\left(a, u_{n}\right)\right)+\operatorname{sc} p\left(\left(a, u_{n}\right),(a, u)\right) \\
& =p\left(a_{n}, a\right) u_{n}+p(a, o)\left|u_{n}-u\right| \rightarrow 0 .
\end{aligned}
$$

If $r x=\mathbf{0}$, then, by $(12), F p\left(x_{n}, x\right)=p\left(\alpha\left(x_{n}\right), o\right) g\left(x_{n}\right)$. We shall consider two cases separately.

Case 1: $x \in U$. Then $F p\left(x_{n}, x\right) \rightarrow p(\alpha(x), o) g(x)=0$ as $\alpha(x)=o$ or $g(x)=0$.

Case 2: $x \in X \backslash U$. Then $F p\left(x_{n}, x\right) \rightarrow 0$, because $g\left(x_{n}\right)=0$ for all but finitely many indices $n$. 
Corollary. Formula (21) defines a map $F: \mathrm{S}(A) \rightarrow \mathrm{S}(X)$ which is linear and has the following properties:

(23) $\|F p\| \leq\|p\| ; p \leq q$ implies $F p \leq F q$,

(24) if $p_{n}(a, b) \rightarrow p(a, b) \forall a, b \in A$, then $F p_{n}(x, y) \rightarrow F p(x, y)$

$$
\begin{array}{r}
F(\mathrm{P}(A)) \subseteq \mathrm{P}(X) ; F(\mathrm{Pc}(A)) \subseteq \mathrm{Pc}(X) ; F p(a, b)=p(a, b) \\
\text { for } a, b \in A .
\end{array}
$$

2.3. Now we shall correct the operator $F$ of Corollary 2.2 in order to get a linear extension operator carrying admissible metrics on $A$ into admissible metrics on $X$. The correction is based on the following lemma due to Lauri Leppik:

LEMMA. Let $r$ be a continuous retraction from a space $Z$ onto its closed subset $Y$. Then there exists a pseudometric $q \in \operatorname{Pbc}(Z)$, with $q \mid Y \times Y=0$, such that if $p \in \operatorname{M}(Y)[p \in \operatorname{Md}(Y)]$ and if

$$
d(x, y)=p(r(x), r(y))+t q(x, y), \quad t>0,
$$

then $d \in \operatorname{M}(Z)[d \in \operatorname{Md}(Z)]$.

Pro of. Let $\ell_{\infty}(Z)$ denote the Banach space of all bounded real functions defined on $Z$ with the supremum norm. Pick a $\delta \in \operatorname{Mab}(Z)$, and define $\Phi: Z \rightarrow \ell_{\infty}(Z)$ by the formula

$$
\Phi(y)(z)=\delta(y, r y) \delta(y, z), \quad y, z \in Z .
$$

For all $x, y, z \in Z$ we have

$$
\begin{aligned}
|\Phi(y)(z)-\Phi(x)(z)|= & \mid \delta(y, r y)(\delta(y, z)-\delta(x, z)) \\
& -\delta(x, z)(\delta(x, r x)-\delta(y, r y)) \mid \\
\leq & \delta(y, r y) \delta(x, y)+\|\delta\|(\delta(x, y)+\delta(r x, r y)) .
\end{aligned}
$$

Hence

$$
\|\Phi(y)-\Phi(x)\| \leq(2 \delta(x, y)+\delta(r x, r y))\|\delta\| .
$$

The required $q$ is defined by

$$
q(x, y)=\|\Phi(x)-\Phi(y)\|=\sup _{z}|\Phi(x)(z)-\Phi(y)(z)| .
$$

From (32) and from the boundedness of $\delta$ it follows that $q \in \operatorname{Pbc}(Z)$. By (31), $\Phi \mid Y=0$, therefore $q \mid Y \times Y=0$.

If $p \in \mathrm{M}(Y)$, then $d(x, y)=0$ implies $r x=r y$ and $q(x, y)=0$, whence

$$
\delta(x, r x) \delta(x, z)=\delta(y, r y) \delta(y, z) \quad \text { for all } z \in Z .
$$

If $r x \neq x$ or $r y \neq y$, then substituting, respectively, $z=y$ or $z=x$ gives $x=y$. Otherwise $x=r x=r y=y$. Thus $d$ is a metric. 
Remember the definition of $d$, and let

$$
y, y_{n} \in Z, \quad d\left(y_{n}, y\right) \rightarrow 0 .
$$

Then, obviously,

$$
q\left(y_{n}, y\right) \rightarrow 0
$$

and $p\left(r y_{n}, r y\right) \rightarrow 0$. If we assume that $p \in \operatorname{Md}(Y)$ and remember that $\delta \in \mathrm{Ma}(Z)$, we also get

$$
\delta\left(r y_{n}, r y\right) \rightarrow 0
$$

$1^{\circ}$ If $y \in Y$, then $\Phi(y)=0$ and

$$
q\left(y_{n}, y\right)=\sup _{z}\left|\Phi\left(y_{n}\right)(z)\right| \geq \delta\left(y_{n}, r y_{n}\right)^{2} .
$$

Hence, by $(35), \delta\left(y_{n}, r y_{n}\right) \rightarrow 0$. By (36) and the fact that $r y=y$, we get $\delta\left(y_{n}, y\right) \rightarrow 0$.

$2^{\circ}$ If $y \in X \backslash Y$, then $\delta(r y, y)>0$ and

$$
\begin{aligned}
q\left(y_{n}, y\right) & =\sup \left|\Phi\left(y_{n}\right)(z)-\Phi(y)(z)\right| \\
& \geq\left|\Phi\left(y_{n}\right)\left(y_{n}\right)-\Phi(y)\left(y_{n}\right)\right|=\delta(y, r y) \delta\left(y, y_{n}\right) .
\end{aligned}
$$

Hence, by $(35), \delta\left(y_{n}, y\right) \rightarrow 0$.

We have shown that, in either case, the assumption (34) implies $\delta\left(y_{n}, y\right)$ $\rightarrow 0$. Since $\delta \in \operatorname{Ma}(Z)$, this means that $d \in \operatorname{Md}(Z)$.

Now we are ready to prove our main results.

TheOrem. Assume that $A$ is an NR of $X$ and $A$ contains at least two distinct points $a_{0}, b_{0}$. Then there exists a linear operator

$$
G: \mathrm{S}(A) \rightarrow \mathrm{S}(X)
$$

such that, for every $p \in \mathrm{S}(A), G p$ is an extension of $p$ with $p \geq 0$ implying $G p \geq 0$, and such that if $\mathrm{K}$ is one of the symbols $\mathrm{P}, \mathrm{M}, \mathrm{Pc}, \mathrm{Pb}, \mathrm{Mc}, \mathrm{Ma}$, $\mathrm{Md}$, then $G(\mathrm{~K}(A)) \subseteq \mathrm{K}(X)$. Moreover, $G$ is continuous with respect to the uniform convergence of functions as well as with respect to their pointwise convergence. The operator $G$ can be defined by the formula

$$
G p(x, y)=F p(x, y)+p\left(a_{0}, b_{0}\right) q(x, y),
$$

where $F$ is the operator of Corollary 2.2 and $q$ is the pseudometric of Lemma 2.3 with $Z=\operatorname{sc} X$ and $Y=\operatorname{sc} A$.

Proof. $G$ is a linear operator as the sum of two linear operators. From Corollary 2.2 and the last lemma it follows that $G(\mathrm{~K}(A)) \subseteq \mathrm{K}(X)$, that $p \geq 0$ implies $G p \geq 0$, and that $\|G(p)\| \leq(1+\|q\|)\|p\|$, which yields the continuity with respect to the uniform convergence. 
Finally, if $p, p_{n} \in \mathrm{S}(A)$, and $p_{n}(a, b) \rightarrow p(a, b)$ for every $a, b \in A$, then

$$
\begin{aligned}
G p_{n}(x, y) & =\operatorname{sc} p_{n}(r x, r y)+p_{n}\left(a_{0}, b_{0}\right) q(x, y) \\
& \rightarrow \operatorname{sc} p(r x, r y)+p\left(a_{0}, b_{0}\right) q(x, y) \\
& =G p(x, y) \quad \text { for all } x, y \in X .
\end{aligned}
$$

COROLlARY. If $A$ is a nondegenerate ANR, then, for every metrizable space $X$ containing $A$ as a closed subset, there is an extension operator with the properties stated in the theorem above.

This is a direct consequence of Proposition 2.2 and the last theorem.

Definition. Let $A$ be a closed subset of $X$. An additive and positivehomogeneous operator $T: \operatorname{Pc}(A) \rightarrow \operatorname{Pc}(X)$ such that $T p \mid A \times A=p$ for $p \in$ $\mathrm{Pc}(A)$ and $T(\mathrm{Ma}(A)) \subseteq \mathrm{Ma}(X)$ is called a regular extensor if $T$ is monotone (i.e., $p \leq q$ implies $T p \leq T q$ ) and continuous both with respect to the uniform convergence of pseudometrics and with respect to their pointwise convergence. A metrizable topological space $X$ is said to have the ARE property if, for every metrizable space $Y$ containing $X$ as a closed subspace, a regular extensor $T: \operatorname{Pc}(X) \rightarrow \operatorname{Pc}(Y)$ exists.

The last corollary says, in particular, that all nondegenerate ANR's have the ARE property. The converse is not true (see $\S 3$ ).

2.4. Example: applying the sc to spheres in normed spaces. Let $X$ be a normed linear space over the reals, $B$ a closed ball in $X, S=\operatorname{bd} B$ the sphere, $o \in S$ a point which will be regarded as base point for the sc operation, and let 0 stand both for the zero vector and the number zero.

ThEOREM. There is a homeomorphism $h$ of $\operatorname{sc} S$ onto $B$ such that $h \mid S=$ id and $h(\mathbf{0})=o$.

Observe that there is no loss of generality in assuming that $B=\{x$ : $\|x-e\| \leq 1\}$, the ball of radius 1 centered at $e$ with $\|e\|=1$, and that $o=0$. Recall that $y \in S$ is said to be a rotundity point of $S$ if, for every $x \in S \backslash\{y\}$ and for every $0<t<1$, the point $t y+(1-t) x$ is not in $S$.

First we establish a special case of the Theorem:

Proposition. If 0 is a rotundity point of $S=\{x:\|x-e\|=1\}$, then the map $g: \operatorname{sc} S \rightarrow B$ given by the formula $g(a, t)=$ ta carries homeomorphically sc $S$ onto $B$, and $g \mid S=\mathrm{id}, g(\mathbf{0})=0$.

Proof. From the rotundity assumption it follows that $g$ is a bijection of sc $S$ onto $B$; obviously $g(S)=S, g(\mathbf{0})=0$. The continuity of $g$ is a direct consequence of Proposition 2.1. The same yields the continuity of $g^{-1}$ at the point 0 .

To prove that $g^{-1}$ is continuous at other points of $B$ assume that $x_{n} \rightarrow$ $x \neq 0$ in $B$ and denote by $\left(y_{n}\right)$ an arbitrary subsequence of $\left(x_{n}\right)$. Pick 
$b, b_{n} \in S$ and $t, t_{n} \in \mathbf{I}$ so that $y_{n}=t_{n} b_{n}$ and $x=t b$. Select a subsequence $\left(y_{n}^{\prime}\right)$ of $\left(y_{n}\right)$ such that the corresponding subsequence $\left(t_{n}^{\prime}\right)$ is convergent, say $t_{n}^{\prime} \rightarrow t^{\prime} \in \mathbf{I}$. Since $t_{n} b_{n} \rightarrow x \neq 0$, and $\left\|b_{n}\right\| \leq 2$ for all $n$, we conclude that $t^{\prime} \neq 0$. Since $S$ is closed, $b_{n}^{\prime}=y_{n}^{\prime} / t_{n}^{\prime} \rightarrow x / t^{\prime}=\left(t / t^{\prime}\right) b \in S$. Therefore $t^{\prime}=t$. Hence

$$
g^{-1}\left(y_{n}^{\prime}\right)=\left(b_{n}^{\prime}, t_{n}^{\prime}\right) \rightarrow(b, t)=g^{-1}(x) \quad \text { in sc } S .
$$

Thus every subsequence of $\left(g^{-1}\left(x_{n}\right)\right)$ admits a subsequence converging to $g^{-1}(x)$, whence $g^{-1}\left(x_{n}\right) \rightarrow g^{-1}(x)$, which is the continuity of $g^{-1}$ at $x$.

It is well known that there exist Banach spaces whose spheres have no rotundity points. However, the following is true:

Lemma. If $(X,\|\cdot\|)$ is a normed space, $S_{1}=\{x:\|x\|=1\}$ the unit sphere, and $e \in S_{1}$, then there is an equivalent norm $|\cdot|$ for $X$ such that $|e|=1$ and $e$ is a rotundity point for the new sphere $S_{2}=\{x:|x|=1\}$.

Pro of. Let $f$ be a continuous linear functional defined on $X$ such that $f(e)=1$. The required norm can be defined by

$$
|x|=\left[f(x)^{2}+\|x-f(x) e\|^{2}\right]^{1 / 2} .
$$

Proof of the Theorem. Let $|\cdot|$ and $e$ be those of the Lemma. Define $B_{0}=\{x:|x-e| \leq 1\}$ and $S_{0}=\{x:|x-e|=1\}$. Obviously 0 is a rotundity point of $S_{0}$. Let $f: X \rightarrow X$ be the homeomorphism acting affinely on each ray emanating from $e$, and carrying $B_{0}$ onto $B$, i.e., $f(e+y)=e+|y| y /\|y\|$ (with the convention $0 / 0=0$ ). Let $g$ be the homeomorphism of the Proposition but applied to the sphere $S_{0}$ rather than $S$. Since $S$ and $S_{0}$ are homeomorphic, they may be regarded as the same topological space equipped with two different admissible bounded metrics. Therefore, by Theorem 2.1, there is a homeomorphism $i$ of sc $S$ onto sc $S_{0}$ such that $i(\mathbf{0})=\mathbf{0}, i(S)=S_{0}$. Now, the formula $h=\left(f \mid B_{0}\right) \circ g \circ i$ defines the required homeomorphism of sc $B$ onto $B$.

\section{Hats and multihats. ARE property does not imply ANR}

3.1. First we define the hat operation: the adjoining of a single squeezed cone over a subset of the space. We shall deal with pointed pairs $(X, A, o)$, where $X, A$ are sets and $o \in A \subseteq X$.

Definition. Given $(X, A, o)$ and a pseudometric $d \in \mathrm{P}(X)$, we let

$$
\text { hat }(X, A, o)=X \cup \operatorname{sc} A \text {, }
$$

where the set $A \subseteq X$ is identified with the subset $A$ of $\operatorname{sc} A$, and

$$
\text { hat } d=\operatorname{sc} d \mid(X \cup \operatorname{sc} A) \times(X \cup \operatorname{sc} A) .
$$

If $X$ is a metrizable topological space, then hat $(X, A, o)$ will be regarded as a topological space with the topology inherited from sc $X$; therefore, by 
Theorem 2.1,

$$
\text { hat } d \in \operatorname{Mab}(\text { hat }(X, A, o)) \quad \text { for every } d \in \operatorname{Mab}(X) \text {. }
$$

Observe that if $A=X$, then hat $(X, A, o)=\operatorname{sc} X$; if $A=\{o\}$, then hat $X=X$. In the sequel we shall assume that $A$ admits at least two distinct points. It is clear that hat $d \mid X \times X=d$. Moreover, (12) and Definition 2.1 yield the explicit formula

(14) $\operatorname{hat} d((a, t), x)=(1-t) d(x, o)+t d(x, a) \quad$ for $(a, t) \in \operatorname{sc} A, x \in X$.

3.2. In this section we shall define the multihat operation: the adjoining of a family of hats. We assume that $X$ is a set and $\mathcal{A}=\left[\left(A_{\lambda}, o_{\lambda}\right)\right]_{\lambda \in \Lambda}$ an indexed family of pointed subsets of $X$.

Definition. Given a pair $(X, \mathcal{A})$ and a $d \in \mathrm{P}(X)$, we let (21) $\operatorname{mht}(X, \mathcal{A})=X \cup \bigcup_{\lambda \in \Lambda} C_{\lambda}, \quad$ where $C_{\lambda}=\left[\operatorname{sc}\left(A_{\lambda}, o_{\lambda}\right) \backslash A_{\lambda}\right] \times\{\lambda\}$.

More conveniently, but with less precision, we may ignore the factors $\{\lambda\}$ in the last formula, remembering instead that the sets $C_{\lambda}$ are pairwise disjoint (even in the case where the members in the family $\mathcal{A}$ are repeated!). Taking this into account we define

$$
\begin{aligned}
& \operatorname{mht} d \mid \operatorname{hat}\left(X, A_{\lambda}, o_{\lambda}\right) \times \operatorname{hat}\left(X, A_{\lambda}, o_{\lambda}\right) \\
& =\operatorname{hat} d \mid \operatorname{hat}\left(X, A_{\lambda}, o_{\lambda}\right) \times \operatorname{hat}\left(X, A_{\lambda}, o_{\lambda}\right) \quad \text { for } \lambda \in \Lambda,
\end{aligned}
$$

and

$$
\begin{aligned}
& \operatorname{mht} d((a, u),(b, v)) \\
& =d(a, b) u v+d\left(a, o_{\mu}\right) u(1-v)+d\left(b, o_{\lambda}\right)(1-u) v \\
& \quad+d\left(o_{\lambda}, o_{\mu}\right)(1-u)(1-v) \quad \text { if }(a, u) \in C_{\lambda},(b, v) \in C_{\mu}, \lambda \neq \mu .
\end{aligned}
$$

Proposition. The function $\operatorname{mht} d: \operatorname{mht}(X, \mathcal{A}) \times \operatorname{mht}(X, \mathcal{A}) \rightarrow \mathbb{R}$ is a pseudometric and $\operatorname{mht} d \in \mathrm{M}(\operatorname{mht}(X, \mathcal{A}))$ if $d \in \mathrm{M}(X)$.

Proof. Since the triangle inequality involves three points, it is enough to consider the case where $\mathcal{A}=\left[\left(A_{\lambda}, o_{\lambda}\right)\right]_{\lambda \in\{1,2,3\}}$. Clearly, then $\operatorname{mht}(X, \mathcal{A})$ can be regarded as a subset of

$$
\operatorname{sc}\left(\operatorname{sc}\left(\operatorname{sc}\left(X, o_{1}\right), o_{2}\right), o_{3}\right),
$$

and it is easily seen that, with this identification,

$$
\operatorname{mht} d=\operatorname{sc}_{3} \mathrm{sc}_{2} \mathrm{sc}_{1} d \mid \operatorname{mht}(X, \mathcal{A}) \times \operatorname{mht}(X, \mathcal{A}) .
$$

This combined with Theorem 2.1 yields the assertion.

Easy consequences of the properties of the sc operation discussed in Sect. 2.1 are summarized in the following: 
Theorem. The operator mht $: \mathrm{P}(X) \rightarrow \mathrm{P}(\operatorname{mht}(X, \mathcal{A}))$ is additive, positive-homogeneous, monotone (i.e., $p \leq q$ implies $\operatorname{mht} p \leq \operatorname{mht} q$ ) and continuous with respect to the pointwise convergence of pseudometrics and with respect to their uniform convergence as well; moreover, $\| \operatorname{mht} p-$ $\operatorname{mht} q\|\leq\| p-q \|$ for $p, q \in \mathrm{P}(X), \operatorname{mht} d \mid X \times X=d$, and $\operatorname{mht} d \mid \operatorname{sc} A_{\lambda} \times$ $\operatorname{sc} A_{\lambda}=\operatorname{sc}\left(d \mid A_{\lambda} \times A_{\lambda}\right), \lambda \in \Lambda$.

3.3. We shall introduce a topology on the multihat $\operatorname{mht}(X, \mathcal{A})$, for $X$ being a metrizable topological space.

Theorem. Assume that $X$ is a metrizable topological space and $\mathcal{A}=$ $\left[\left(A_{\lambda}, o_{\lambda}\right)\right]_{\lambda \in \Lambda}$, where $o_{\lambda} \in A_{\lambda}$ for $\lambda \in \Lambda$. Then the topology of the metric space $(\operatorname{mht}(X, \mathcal{A}), \operatorname{mht} d)$ does not depend on the choice of the metric $d$ in $\operatorname{Mab}(X)$.

The topology described by the theorem will be referred to as the topology of the multihat mht $(X, \mathcal{A})$.

Pro of. Let $d \in \operatorname{Mab}(X)$. Define: $Y=\operatorname{mht}(X, \mathcal{A}), Y_{\lambda}=\operatorname{sc} A_{\lambda} \backslash A_{\lambda}=$ hat $\left(X, A_{\lambda}, o_{\lambda}\right) \backslash X$, and $\delta=\operatorname{mht} d$. By (14), (22) and (23) we have

(31) $\operatorname{dist}_{\delta}\left(z, Y \backslash Y_{\lambda}\right) \geq \min [t, 1-t] d\left(a, o_{\lambda}\right)>0 \quad$ for each $z=(a, t) \in Y_{\lambda}$.

Thus each $Y_{\lambda}$ is open in the metric space $(Y, \delta)$.

The theorem immediately follows from the last statement, from the fact that $\delta$ extends $d$ and from the next lemma, which together characterize the convergence in the metric space $(Y, \delta)$.

Lemma. Let $y_{n}=\left(x_{n}, t_{n}\right) \in Y_{\lambda(n)}$ for $n \in \mathbb{N}\left(x_{n} \in A_{\lambda(n)} \subseteq X\right)$, and let $y \in Y$. Then

(i) $y_{n} \rightarrow y=(x, t) \in Y_{\lambda}$ iff $\lambda(n)=\lambda$ for all but finitely many $n$ 's and $t_{n} \rightarrow t, x_{n} \rightarrow x$

(ii) $y_{n} \rightarrow y=x \in X$ iff for every $\varepsilon>0$ each set

$$
\begin{aligned}
& \mathbb{N}_{0}(\varepsilon)=\left\{n: 1-t_{n} \geq \varepsilon \text { and } d\left(o_{\lambda(n)}, x\right) \geq \varepsilon\right\}, \\
& \mathbb{N}_{1}(\varepsilon)=\left\{n: t_{n} \geq \varepsilon \text { and } d\left(x_{n}, x\right) \geq \varepsilon\right\}, \\
& \mathbb{N}_{2}(\varepsilon)=\left\{n: \min \left[d\left(o_{\lambda(n)}, x\right), d\left(x_{n}, x\right)\right] \geq \varepsilon\right\}
\end{aligned}
$$

is finite.

It is easily seen that the finiteness of the sets in (ii) does not depend on the particular choice of the metric $d \in \operatorname{Mab}(X)$, although $d$ appears in their definitions.

Pro of of (i). This is an immediate consequence of (31).

Pro of of (ii). Assume that $n$ is not in $\mathbb{N}_{0}(\varepsilon) \cup \mathbb{N}_{1}(\varepsilon) \cup \mathbb{N}_{2}(\varepsilon)$ and that $\varepsilon \leq 2^{-1}$. Then either $d\left(o_{\lambda(n)}, x\right)<\varepsilon$ and $t_{n}<\varepsilon$, or $d\left(x_{n}, x\right)<\varepsilon$ and $1-t_{n}<\varepsilon$. 
By (14) and (22) we have

$$
\delta\left(y_{n}, x\right)=t_{n} d\left(x_{n}, x\right)+\left(1-t_{n}\right) d\left(o_{\lambda(n)}, x\right) .
$$

Hence $\delta\left(y_{n}, x\right) \leq \varepsilon\|d\|+\varepsilon$. Thus the condition of (ii) implies the convergence $y_{n} \rightarrow x$.

On the other hand, by $(32), \mathbb{N}_{0}(\varepsilon) \cup \mathbb{N}_{1}(\varepsilon) \subseteq\left\{n: \delta\left(y_{n}, x\right) \geq \varepsilon^{2}\right\}$ and

$$
\delta\left(y_{n}, x\right) \geq \min \left[d\left(x_{n}, x\right), d\left(o_{\lambda(n)}, x\right)\right] .
$$

Hence

$$
\mathbb{N}_{0}(\varepsilon) \cup \mathbb{N}_{1}(\varepsilon) \cup \mathbb{N}_{2}(\varepsilon) \subseteq\left\{n: \delta\left(y_{n}, x\right) \geq \varepsilon^{2}\right\} \cup\left\{n: \delta\left(y_{n}, x\right) \geq \varepsilon\right\},
$$

which is finite if $y_{n} \rightarrow x$.

This completes the proof of (ii).

COROLlaRY. Under the assumption of the Theorem, the operator mht : $\mathrm{P}(X) \rightarrow \mathrm{P}(\operatorname{mht}(X, A))$ has the properties:

$\operatorname{mht}(\operatorname{Pbc}(X)) \subseteq \operatorname{Pbc}(\operatorname{mht}(X, \mathcal{A})), \operatorname{mht}(\operatorname{Mab}(X)) \subseteq \operatorname{Mab}(\operatorname{mht}(X, \mathcal{A}))$.

Moreover, if $X$ is compact, then $\operatorname{mht} \mid \operatorname{Pc}(X)$ is a regular extensor.

Theorem 3.3 is due to the referee; the proof presented here is a slight modification of his proof. The theorem stated in the original version of the manuscript was valid only in the case where $\mathcal{A}=\left[\left(Y_{n}, o_{n}\right)\right]_{n \in \mathbb{N}}$, with $Y_{n}$ closed and satisfying the condition (i) of the lemma below for $(Y, p)=(X, d)$.

3.4. Lemma. Let $(Y, p)$ be a metric space, let $\left(Y_{n}\right)$ be a sequence of closed subspaces of $Y$ such that:

(i) $\operatorname{diam} Y_{n} \rightarrow 0$, bd $Y_{n} \backslash \bigcup_{k \in \mathbb{N}}$ int $Y_{k} \neq \emptyset$,

and let $f$ be a map from $Y$ to a metric space $(E, q)$ such that:

(ii) $\operatorname{diam} f\left(Y_{n}\right) \rightarrow 0$,

(iii) $f \mid Y \backslash \bigcup_{k \in \mathbb{N}}$ int $Y_{k}$ is continuous,

(iv) $f \mid Y_{k}$ is continuous for every $k \in \mathbb{N}$.

Then $f$ is continuous.

Proof. Assume that $x_{n} \rightarrow x$ in $Y$. If $x \in \operatorname{int} Y_{k}$ for some $k$, then $f\left(x_{n}\right) \rightarrow f(x)$ by (iv); if $\left(y_{n}\right)$ is a subsequence of $\left(x_{n}\right)$ such that all $y_{n}$ 's are in one set $Y_{k}$ or all of them are in $Y \backslash \bigcup_{k \in \mathbb{N}}$ int $Y_{k}$, then $f\left(y_{n}\right) \rightarrow f(x)$ by (iv) and (iii). Therefore, without loss of generality, we may assume that $x \in Y \backslash \bigcup_{k \in \mathbb{N}}$ int $Y_{k}$ and that $x_{n} \in \operatorname{int} Y_{k(n)}$ where $k(n) \rightarrow \infty$. By (i) we can pick $z_{n} \in \operatorname{bd} Y_{k(n)} \backslash \bigcup_{k \in \mathbb{N}}$ int $Y_{k}$ and then $z_{n} \rightarrow x$ by (i) again. By (iii), $f\left(z_{n}\right) \rightarrow f(x)$, whence, by (ii), $f\left(x_{n}\right) \rightarrow f(x)$.

In the original version of the manuscript the statement of the lemma was incorrect (the second condition in (i)). Its present appearance is due to the referee. 
3.5. Let $\mathbb{P}$ be a closed $r$-dimensional cube, $r \in \mathbb{N}$. Let $\left(K_{n}\right)_{n \in \mathbb{N}}$ be a sequence of pairwise disjoint open Euclidean balls contained in $\mathbb{P}$. Finally, let $A_{n}=\operatorname{bd} K_{n}$ and $o_{n} \in A_{n}$ for $n \in \mathbb{N}$, and $\mathcal{A}=\left[\left(A_{n}, o_{n}\right)\right]$.

TheOREM. The space $\mathbb{X}=\mathbb{P} \backslash \bigcup_{n \in \mathbb{N}} K_{n}$ has the ARE property but is not an ANR.

First, we establish the following

LEMma. The space $\operatorname{mht}(\mathbb{X}, \mathcal{A})$ is homeomorphic to the cube $\mathbb{P}$ and, therefore, is an ANR.

Pr o o f. For each $n \in \mathbb{N}$, let $g_{n}$ be the homeomorphism of Proposition 2.4 carrying $\operatorname{sc}\left(A_{n}, o_{n}\right)$ onto the closed ball $K_{n} \cup A_{n}$, which is the identity on $A_{n}$. Define $f: \operatorname{mht}(\mathbb{X}, \mathcal{A}) \rightarrow \mathbb{P}$ by

$$
f\left|\mathbb{X}=\mathrm{id}_{\mathbb{X}}, \quad f\right| \operatorname{sc} A_{n}=g_{n} .
$$

Applying Lemma 3.4 to $f$ and to $f^{-1}$ (with $Y_{n}=\operatorname{sc} A_{n}$ and with $Y_{n}=$ $K_{n} \cup A_{n}$ ), we get the assertion of our Lemma.

Proof of the Theorem. Assume that $Y$ is a metric space containing $\mathbb{X}$. By Theorem 3.3 the multihats $\operatorname{mht}(\mathbb{X}, \mathcal{A})$ and $\operatorname{mht}(Y, \mathcal{A})$ are metrizable topological spaces. Since $\mathbb{X}$ is a closed (compact) subset of $Y$, we conclude that the ANR space $\operatorname{mht}(\mathbb{X}, \mathcal{A})$ is a closed subspace and a neighborhood retract of $\operatorname{mht}(Y, \mathcal{A})$. By Corollary 2.3, there is a regular extensor $G$ : $\operatorname{Pc}(\operatorname{mht}(\mathbb{X}, \mathcal{A})) \rightarrow \operatorname{Pc}(\operatorname{mht}(Y, \mathcal{A}))$. By Corollary 3.3, the extension operator mht $: \mathrm{P}(\mathbb{X}) \rightarrow \mathrm{P}(\operatorname{mht}(\mathbb{X}, \mathcal{A}))$ restricted to $\mathrm{Pc}(\mathbb{X})$ is also a regular extensor.

Recalling that $Y \subseteq \operatorname{mht}(Y, \mathcal{A})$ we define the required regular extensor $T: \operatorname{Pc}(\mathbb{X}) \rightarrow \operatorname{Pc}(Y)$ by the formula

$$
T p(y, z)=G(\operatorname{mht} p)(y, z) \quad \text { for } y, z \in Y, p \in \operatorname{Pc}(\mathbb{X}) .
$$

Since $\mathbb{X}$ is compact, and $\operatorname{diam} A_{n} \rightarrow 0$, the spheres $A_{n}$ have at least one cluster point, say $x_{0}, \mathbb{X}$ is not locally contractible at $x_{0}$, and therefore (cf. [Bor]) is not an ANR.

3.6. We shall define some other extension constructions related to the sc: chord (attaching a single string), isol (adjoining an isolated point), c (the cone construction), harp (attaching a family of strings).

Given a bi-pointed set $(A, a, \alpha), a, \alpha \in A, a \neq \alpha$, define

$$
\operatorname{chord}(A, a, \alpha)=\operatorname{hat}(A,\{a, \alpha\}, a), \quad \operatorname{chord} p=\operatorname{hat} p \quad \text { for } p \in \mathrm{P}(A) \text {. }
$$

The subset $S=\operatorname{sc}(\{a, \alpha\}, a)$ is referred to as the string attached to the points $a, \alpha$. The point $(\alpha, t) \in S, t \in \mathbf{I}$, will be written in the form

$$
t \alpha \oplus(1-t) a .
$$


The last expression together with the formula (14) and the formula (11) in 2.1 show that the roles of the end-points $a, \alpha$ are symmetric. We define

$$
i \operatorname{sol}(A, a, \alpha)=A \cup\left\{2^{-1} \alpha \oplus 2^{-1} a\right\}, \quad i \operatorname{sol} p=\operatorname{chord} p \mid \operatorname{isol}(A, a, \alpha) \text {. }
$$

We define $\mathrm{c}(A, a, \alpha)=\operatorname{sc}(\operatorname{isol}(A, a, \alpha), o)$ where $o=2^{-1} \alpha \oplus 2^{-1} a$ and c $p=\operatorname{sc}($ isol $p)$.

Given a set $A$ admitting at least two distinct points and a family $\mathcal{B}=$ $\left[\left(a_{\lambda}, b_{\lambda}\right)\right]_{\lambda \in \Lambda}$ of pairs of distinct points of $A$, define $\operatorname{harp}(\mathcal{A}, \mathcal{B})=\operatorname{mht}(A, \mathcal{A})$ with $\mathcal{A}=\left[\left(\left\{a_{\lambda}, b_{\lambda}\right\}, a_{\lambda}\right)\right]_{\lambda \in \Lambda}$ and harp $q=\operatorname{mht} q$. The subsets

$$
S_{\lambda}=\operatorname{sc}\left(\left\{a_{\lambda}, b_{\lambda}\right\}, a_{\lambda}\right)=\left\{t b_{\lambda} \oplus_{\lambda}(1-t) a_{\lambda}: t \in \mathbf{I}\right\}, \quad \lambda \in \Lambda,
$$

of harp $(A, \mathcal{B})$ are referred to as the strings of the harp. Let us note that each string is well-spanned, i.e., it is isometric to the line segment of length equal to the distance between the ends of the string, and, even if all the strings have common ends, they do not touch each other, except at the ends. Of course such a strange harp cannot be placed in any Euclidean space (more generally: in a normed space with rotund norm) but, perhaps, can be played by angels.

\section{The sc construction and related metric extensions as functors}

4.1. The category $\mathcal{S E \mathcal { T }} \mathcal{T}_{1}$ and the construction sc. The objects of $\mathcal{S E} \mathcal{T}_{1}$ are pointed sets $(A, a)$, and morphisms $f:(A, a) \rightarrow(B, b)$ are maps $f: A \rightarrow$ $B$ such that $f(a)=b$. The construction sc is a triple of maps: sc, $\mathrm{sc}^{\prime}, \mathrm{sc}^{\prime \prime}$ acting on objects, morphisms and pseudometrics, respectively, which are defined as follows:

$$
\operatorname{sc}(A, a)=((A \backslash\{a\}) \times \mathcal{J} \cup A, a)
$$

where $\mathcal{J}=(0 ; 1)$, the open interval; if $f:(A, a) \rightarrow(B, b)$, then $\operatorname{sc}^{\prime} f:$ $\operatorname{sc}(A, a) \rightarrow \operatorname{sc}(B, b)$ is defined by

$$
\operatorname{sc}^{\prime} f(y)=f(y), \quad \operatorname{sc}^{\prime} f(x, u)= \begin{cases}(f(x), u) & \text { if } f(x) \neq b, \\ b & \text { if } f(x)=b,\end{cases}
$$

for $y \in A,(x, u) \in(A \backslash\{a\}) \times \mathcal{J}$; for $p \in \mathrm{P}(A, a)$ we let $\mathrm{sc}^{\prime \prime} p=q \in$ $\mathrm{P}(\operatorname{sc}(A, a))$ be defined by

$$
\begin{aligned}
& q(x, y)=p(x, y) \quad \text { if } x, y \in A \\
& q((x, u), y)=(1-u) p(y, a)+u p(y, x), \\
& \begin{array}{r}
q((x, u),(y, v))=2^{-1}[p(x, y)(u+v-|u-v|) \\
\quad+p(x, a)(u-v+|u-v|)+p(y, a)(v-u+|u-v|)] .
\end{array}
\end{aligned}
$$

We note that the pair (sc, sc $^{\prime \prime}$ ) corresponds to the sc defined in Sect. 2.1. The reason for redefining it was to avoid identifications of points of $A \times \mathbf{I}$ and obtain the genuine inclusion $A \subseteq$ sc $A$. 
For every morphism $f:(A, a) \rightarrow(B, b)$ and for every pseudometric $p \in \mathrm{P}(B, b)$ we define $f_{*} p \in \mathrm{P}(A, a)$ by

$$
f_{*} p(x, y)=p(f(x), f(y)) \quad \text { for } x, y \in A .
$$

We shall list some properties of the defined notions which easily follow from the results of Sect. 2.1 and from formulas (41) and (42):

(43) The pair $\left(\mathrm{sc}, \mathrm{sc}^{\prime}\right)$ is a functor on the category $\mathcal{S E \mathcal { T }} \mathcal{T}_{1}$.

(44) If $f:(A, a) \rightarrow(B, b)$ and $q \in \mathrm{P}(B, b)$, then $\operatorname{sc}^{\prime \prime}\left(f_{*} q\right)=\left(\mathrm{sc}^{\prime} f\right)_{*} \mathrm{sc}^{\prime \prime} q$.

(45) If $f:(A, a) \rightarrow(B, b)$ and $p \in \mathrm{P}(A), q \in \mathrm{P}(B)$ are such that $p$ dominates $f_{*} q$ and $\|q\|<\infty\left[\right.$ such that $\left.f_{*} q \leq p\right]$, then $\mathrm{sc}^{\prime \prime} p$ dominates $\left(\mathrm{sc}^{\prime} f\right)_{*} \mathrm{sc}^{\prime \prime} q\left[\left(\mathrm{sc}^{\prime} f\right)_{*} \mathrm{sc}^{\prime \prime} q \leq \mathrm{sc}^{\prime \prime} p\right]$.

(46) If $A, B$ are metrizable topological spaces, and $f: A \rightarrow B$ is a continuous map with $f(a)=b$, then $\operatorname{sc}^{\prime} f: \operatorname{sc}(A, a) \rightarrow \operatorname{sc}(B, b)$ is continuous.

(47) If $A$ and $B$ are equipped with metrics $d$ and $\delta$, respectively, and $f:(A, a) \rightarrow(B, b)$ is Lipschitzian (i.e., $\left.f_{*} \delta \leq C d\right)$, then $\mathrm{sc}^{\prime} f:$ $\mathrm{sc}(A, a) \rightarrow \mathrm{sc}(B, b)$ satisfies the Lipschitz condition with the same constant $C$.

We recall that the topology of $\operatorname{sc}(A, a)$ is the one determined by $\mathrm{sc}^{\prime \prime} d$ where $d$ is an arbitrary admissible bounded metric for $A$. The statements (46) and (47) are immediate consequences of (45) and (45) [...], respectively.

4.2. The category $\mathcal{P} \mathcal{A} \mathcal{I} \mathcal{R}_{1}$ and the construction hat. Objects of $\mathcal{P} \mathcal{A} \mathcal{I} \mathcal{R}_{1}$ are pointed pairs $(X, A, a)$; morphisms $f:(X, A, a) \rightarrow(Y, B, b)$ are maps $f: X \rightarrow Y$ such that $f(A) \subseteq B$ and $f(a)=b$. The construction hat is the triple hat, hat', hat'", where

$$
\begin{aligned}
& \text { hat }(X, A, a)=(X \cup(A \backslash\{a\}) \times \mathcal{J}, A, a) \text {; } \\
& \text { hat' }^{\prime} f(x)=f(x) \text {, } \\
& \text { hat }^{\prime} f(y, u)= \begin{cases}(f(y), u) & \text { if } f(y) \neq b, \\
b & \text { if } f(y)=b ;\end{cases} \\
& \text { hat }^{\prime \prime} p=\operatorname{sc}^{\prime \prime} p \mid \text { hat }(X, A, a), \quad \text { where } \operatorname{sc}^{\prime \prime}: \mathrm{P}(X) \rightarrow \mathrm{P}(\operatorname{sc}(X, a)) \text {. }
\end{aligned}
$$

The pair (hat, hat') is a functor acting on $\mathcal{P} \mathcal{A} \mathcal{I} \mathcal{R}_{1}$.

4.3. The category $\mathcal{S E T}_{2}$ and the constructions: chord, isol, cone. $\mathcal{S E} \mathcal{T}_{2}$ is the category of bi-pointed sets $(A, a, \alpha), a, \alpha \in A, a \neq \alpha$, where the morphisms $f:(A, a, \alpha) \rightarrow(B, b, \beta)$ are maps $f: A \rightarrow B$ such that $f(a)=b$ and $f(\alpha)=\beta$.

The construction chord is the triple chord, chord', chord", where

$$
\begin{aligned}
& \operatorname{chord}(A, a, \alpha)=(A \cup\{t \alpha \oplus(1-t) a: t \in \mathcal{J}\}, a, \alpha) ; \\
& \operatorname{chord}^{\prime} f(x)=f(x),
\end{aligned}
$$




$$
\begin{aligned}
& \text { chord' }^{\prime} f(t \alpha \oplus(1-t) a)=t \beta \oplus(1-t) b ; \\
& \text { chord" }^{\prime \prime} p(x, y)=p(x, y), \\
& \text { chord" }^{\prime \prime} p(t \alpha \oplus(1-t) a, y)=t p(\alpha, y)+(1-t) p(a, y), \\
& \text { chord" }^{\prime \prime}(t \alpha \oplus(1-t) a, u \alpha \oplus(1-u) a)=|t-u| p(a, \alpha) .
\end{aligned}
$$

Clearly, (chord, chord') is a functor acting on $\mathcal{S E T}_{2}$.

The construction isol is the triple isol, isol' ${ }^{\prime}$ isol":

$$
\begin{aligned}
& \text { isol }(A, a, \alpha)=\left(A \cup\left\{o_{A}\right\}, o_{A}\right) \quad \text { where } o_{A}=2^{-1} \alpha \oplus 2^{-1} a \\
& \text { isol' }^{\prime}(x)=f(x) \text {, } \\
& \text { isol' }^{\prime}\left(o_{A}\right)=o_{B} \quad \text { for } f:(A, a, \alpha) \rightarrow(B, b, \beta) \text {; } \\
& \text { isol" } p(x, y)=p(x, y) \text {, } \\
& \text { isol" } p\left(y, o_{A}\right)=2^{-1}(p(y, \alpha)+p(y, a)) \text {. }
\end{aligned}
$$

The pair (isol, isol') is a functor from the category $\mathcal{S E} \mathcal{T}_{2}$ to $\mathcal{S E \mathcal { T }} \mathcal{T}_{1}$.

Finally, the cone construction is the triple $\mathrm{c}, \mathrm{c}^{\prime}, \mathrm{c}^{\prime \prime}$, where $\left(\mathrm{c}, \mathrm{c}^{\prime}\right)$ is the functor from $\mathcal{S E T}_{2}$ to $\mathcal{S E} \mathcal{T}_{1}$ which is the composition of the functors (isol, isol') and $\left(\mathrm{sc}, \mathrm{sc}^{\prime}\right) ; \mathrm{c}^{\prime \prime} p=\mathrm{sc}^{\prime \prime}\left(\right.$ isol $\left.^{\prime \prime} p\right)$.

5. Problems and comments. The word "linear", when applied to cones $\mathrm{P}(X), \mathrm{M}(X)$, means "additive and positive-homogeneous". The word "compactum" means a "metrizable compact space".

The problems are arranged according to the sections they are related to. The crucial one for our subject is Problem 221.

To keep within the framework of Banach spaces, we ask most of the questions about pseudometrics for compacta, although these questions admit analogues for general metrizable spaces.

To Section 1.2: For a set $A$ with card $A \geq 2$, the positive multiples of the pseudometrics $p_{B}$ defined by (29) are called in the literature "Hamming semimetrics" (we say: Hamming pseudometrics). Lemma 1.2 is due to Deza [De]. It says that, if $2 \leq \operatorname{card} A \leq 4$, then every $d \in \mathrm{P}(A)$ is the sum of a certain number of Hamming pseudometrics. This is not so when card $A \geq 5$. The simplest example is $A=\{1,2,3,4,5\}$; the metric $d \in \mathrm{M}(A)$ defined by $d(i, j)=3+(-1)^{i+j}$ for $i \neq j, d(i, i)=0$ cannot be written as a sum of Hamming pseudometrics (it is easily seen that subtracting from $d$ any Hamming pseudometric violates a triangle inequality). Clearly, $d$ also cannot be expressed as $p+q$ with $p, q \in \mathrm{P}(A) \backslash \mathrm{M}(A)$. For information on Hamming pseudometrics and their relation to the problem of isometric embeddings of metric spaces in $L_{1}$ see [AM], [Av], [D], [De], [AD], [K], [Ke].

Problem 121. Describe all edge vectors of the polyhedral cone $\mathrm{P}_{n}=$ $\mathrm{P}(A)$ with $\operatorname{card} A=n$. 
As far as I know the question has been answered only for small values of $n$.

\section{To Section 1.4:}

Problem 141. Let $X$ be a compactum. Investigate isomorphism properties of the Banach space $E(X)$ generated in the space $C(X \times X)$ by $\operatorname{Pc}(X)$. Does $E(X)$ always have the approximation property or a basis?

By a Dugundji operator of a compact pair $(Y, Z)$ we mean a bounded linear operator $T: C(Z) \rightarrow C(Y)$ such that $T f \mid Z=f$ for $f \in C(Z)$.

Problem 142. Given a compactum $A$, let $X=\operatorname{sc} A$. Does the operator sc $: \operatorname{Pc}(A) \rightarrow \operatorname{Pc}(X)$ extend to a Dugundji operator from $C(A \times A)$ into $C(X \times X) ?$

To Section 2.3: We note that, in the compact case, the proofs of the main results are much simpler (cf. [B]).

Problem 231. Does every compact pair $(X, A)$ admit a regular extensor? Do there exist norm-continuous linear extending operators from $\mathrm{Ma}(A)$ into $\mathrm{Ma}(X)$ ?

Conjecture. The answer to the first question is "no", to the second "yes".

Problem 232. For a compactum $X$ describe all the continuous linear functionals on $\operatorname{Pc}(X)$. Are they represented as integrals of $p(x, y)$ with respect to certain Borel measures on $X \times X$ ?

Problem 233. For a compact pair $(X, A)$, card $A \geq 2$, every normcontinuous linear extending operator from $\operatorname{Pc}(A)$ into $\operatorname{Pc}(X)$ can be "perturbed" to get an extension operator carrying $\mathrm{Ma}(A)$ into $\mathrm{Ma}(X)$. (Use the trick in the proof of Theorem 1 in $[\mathrm{KN}]$ for a fixed metric.) Can this be done in the noncompact case?

We note here that the proof of Leppik's Lemma is a modification of the [KN] trick.

Problem 234. Assume that $(X, A)$ is a compact pair which admits a regular extensor, and $\delta \in \mathrm{Ma}(X)$ is a fixed metric. Does there exist a regular extensor $T: \operatorname{Pc}(A) \rightarrow \operatorname{Pc}(X)$ and a constant $c>0$ such that $p \leq \delta \mid A$ implies $T p \leq c \delta$ ?

Problem 235. Let $A$ and $B$ be compacta. If $\operatorname{sh} B=\operatorname{sh} A$ and $A$ has the ARE property, does $B$ have it? (The symbol sh denotes the Borsuk shape [Bors]). 


\section{To Section 3.6:}

Problem 361. Given a pointed metric space $(X, o)$ and a metrizable pair $(B, A)$ with $A$ closed, consider the reduced Cartesian product $Z=$ $(B \times X)_{A}=A \cup(B \backslash A) \times X$, with the topology defined in [BP], p. 25 (cf. also [AS], p. 316). The space $B$ is regarded as a subspace of $Z$ by means of the identification: $a \rightarrow a$ for $a \in A, x \rightarrow(x, o)$ for $x \in B \backslash A$. Write an explicit formula for a metric $\delta \in \mathrm{Ma}(Z)$ which extends a given metric $d \in \mathrm{Ma}(B)$. Can this be done so as to obtain an extension construction?

To Section 4.1: Of course $\mathrm{P}$ is a functor from $\mathcal{S E \mathcal { T }} 1$ to $\mathcal{S E \mathcal { T }}$ (the category of sets): for $f: A \rightarrow B, \mathrm{P} f=f_{*}: \mathrm{P}(B) \rightarrow \mathrm{P}(A)$. It might be of interest to study the interaction of the functor $\mathrm{P}$ with the extending functor and construction sc and with related ones.

Finally, we note that similar problems to those on linear extending of metrics can be stated in the equivariant context: $1^{\circ}$ for spaces with a group action; $2^{\circ}$ for pointed spaces with a group action fixing the base point.

For a Banach space $X$ let $\mathrm{Na}(X)$ be the cone of all admissible norms on $X$ equipped with the topology induced by the metric

$$
\delta(p, q)=\sup \{|\log p(x)-\log q(x)|: x \in X \backslash\{0\}\}, \quad p, q \in \mathrm{Na}(X) .
$$

Problem A. Let $X$ be a Banach space and $Y$ its closed linear subspace. Does there exist a [continuous] linear operator $\Phi: \mathrm{Na}(Y) \rightarrow \mathrm{Na}(X)$ which extends norms?

Problem B. Let $X$ be a Banach space. Does there exist a retraction $r: \operatorname{Mainv}(X) \rightarrow \mathrm{Na}(X)$ which is continuous in any sense? The symbol $\operatorname{Mainv}(X)$ denotes the set of admissible invariant metrics for $X$.

\section{References}

[AS] R. D. Anderson and R. Schori, Factors of infinite-dimensional manifolds, Trans. Amer. Math. Soc. 142 (1969), 315-330.

[AD] P. Assouad and M. Deza, Metric subspaces of $L^{1}$, Publ. Math. Orsay 3 (1982), 47 pp.

[Av] D. Avis, Hypermetric spaces and the Hamming cone, Canad. J. Math. 33 (1981), 795-802.

[AM] D. Avis and Mutt, All the facets of the six-point Hamming cone, European J. Combin. 10 (1989), 309-312.

[B] C. Bessaga, Functional analytic aspects of geometry. Linear extending of metrics and related problems, in: Progress in Functional Analysis, Proceedings of the Peniscola Meeting 1990 on the occasion of the 60th birthday of Professor M. Valdivia, North-Holland Math. Stud. 170, North-Holland, Amsterdam 1992, $247-257$. 
[BP] C. Bessaga and A. Pełczyński, Selected Topics in Infinite-Dimensional Topology, PWN, Warszawa 1975.

[Bo] K. Borsuk, Über Isomorphie der Funktionalräume, Bull. Internat. Acad. Polon. Sér. A 1933, 1-10.

[Bor] —, Theory of Retracts, PWN, Warszawa 1967.

[Bors] - , Theory of Shape, PWN, Warszawa 1975.

[D] M. Deza (Tylkin), On Hamming geometry of unitary cubes, Dokl. Akad. Nauk SSSR 134 (1960), 1037-1040 (in Russian).

[De] - Matrices des formes quadratiques non négatives pour des arguments binaires, C. R. Acad. Sci. Paris Sér. A-B 277 (1973), A873-A875.

[Du] J. Dugundji, An extension of Tietze's theorem, Pacific J. Math. 1 (1951), 353367.

[H] F. Hausdorff, Erweiterung einer Homöomorphie, Fund. Math. 16 (1930), 353360.

[K] J. B. Kelly, Metric inequalities and symmetric differences, in: Inequalities II, Academic Press, New York 1970, 193-212.

[Ke] - , Hypermetric spaces, in: The Geometry of Metric and Linear Spaces, Lecture Notes in Math. 490, Springer, 1975, 17-31.

[L] J. Luukkainen, Extension of spaces, maps, and metrics in Lipschitz topology, Ann. Acad. Sci. Fenn. Ser. A I Math. Dissertationes 17 (1978), 1-62.

[N] Nguyen To Nhu, Extending metrics uniformly, Colloq. Math. 43 (1980), 91-97.

[KN] Nguyen Van Khue and Nguyen To Nhu, Two extensors of metrics, Bull. Acad. Polon. Sci. 29 (1981), 285-291.

[T] H. Toruńczyk, A short proof of Hausdorff's theorem on extending metrics, Fund. Math. 77 (1972), 191-193.

INSTITUTE OF MATHEMATICS

WARSAW UNIVERSITY

BANACHA 2

02-097 WARSZAWA, POLAND

Received 25 March 1991;

in revised form 12 December 1991, 12 May 1992 and 10 August 1992 